\title{
Digitized Decision-Making System Research for Warehouse Planning Using Navigation Patterns Based on Visual LISP
}

\author{
http://dx.doi.org/10.3991/ijoe.v9i5.2975 \\ H. Yao, Y. D. Fang, Y. Cao \\ School of Mechatonic Engineering, Xi’an Technological University, Xi’an, China,
}

\begin{abstract}
To solve the problem of decision making for the planning and construction of warehouse area, a digitized decision-making system is developed based on Auto CAD with the development tools of Visual LISt processor (LISP) in the paper. Using navigation patterns, a user is guided to accomplish the analysis, estimates, and the calculation of construction costs for various types of facilities planning and layout in warehouse area. The modular design method is utilized to build the system function model. The system includes analysis and estimates module, statistics and construction cost module and system management module. With the interface program between the system management module and the structured query language (SQL) database, the functional models are connected with the database dynamically. The man-machine interface established with Dialog Control Language is easy to use and accords with industry habits. All engineering parameters from national standards and industry standards are reliable and digitalized to establish parameter databases. The interface between Auto computer-aided design (CAD) and the parameter databases is developed to call the data in the parameter databases from AutoCAD. Consequently, the efficiency and quality of warehouse planning can be improved. The system can also be extended to form a complete and standard digitized decision-making CAD system and improve the scientific rationality of the planning decisions for warehouse area.
\end{abstract}

Index Terms-CAD, decision making system, digitized, Visual LISP, warehouse

\section{INTRODUCTION}

With the rapid development of the current socioeconomic, manufacturing, commerce, and the greatly enhance of the human material civilization, products, raw materials, a large demand, and the amount of logistics is also increasing [1][2]. At present, modern manufacturing enterprise environment has undergone great changes, the degree of automation of the production equipment has been increased rapidly, the life cycle of the product has been greatly shortened and the delivery time has become a major competitive factor [3]. In order to meet the material higher requirements of production line, materials in the production process are inevitably required to be supplied on time and accurately. Especially for small-lot and multi-species manufacturing in flexible storage DWG files and establish the relationship between the drawing element and the object represented and the dynamic association between the graphics library and the manufacturing enterprise, a flexible management is also proposed for materials warehouse [4]. Meanwhile, logistics has proceeded beyond a single industry and become a principal condition for economic competition [5]. To improve the efficiency of storage and transportation of goods, the warehouse is widely used in different kinds of fields. The current warehouses, which are often operated manually, usually include palletizing robots, carton flow order picking systems, automated guided vehicles, rotary storage cabinets, and automated storage and retrieval systems (AS/RSs) [6][7]. The main shortcoming of the current warehouse is that the efficiency of its storage and retrieval systems is very low, which is also a bottleneck to restrict the development of automated warehouse [8]. Now there are some problems in the material warehouse, for example, storage areas are not clearly demarcated; the materials working routes are convection or roundabout; the supplies efficiency has been seriously affected. Due to these problems, the computer-aided decision-making system needs to support all aspects of the planning and construction of the warehouse and enhance the scientificity of the macroplanning and improve the accuracy and efficiency of estimates.

\section{FUNCTIONAL ANALYSIS}

A complete logistics and distribution center or warehouse area contains a wide range of facilities and equipments, which are divided into three categories: logistics operation area equipments, auxiliary area facilities and plant construction facilities [9]. With the unified deployment and information management of digital construction system considered and with the warehouse, ancillary buildings, logistics routes and various types of pipelines as the research objects, this paper aims to design an assistant decision-making system based on warehouse area planning scheme and drawings. The system has three functions: the first is analysis and estimates function that includes analyzing and estimating area or volume for warehouse, parking area, roads and green areas, etc. In addition, it also can estimate the length of logistics routes, roads, underground pipelines and supply lines and so on. The second is statistics and construction cost function, it aims to generate the construction costs preplan. In AutoCAD, the system can get each basic drawing element data from the entire SQL database. Based on these, the system calculates the construction costs and meets demands of the engineering staff to query, statistics, intelligently decide and process 
the data of warehouse, logistics route and others. The last is auxiliary function, both estimates function and data management function cannot achieve successfully without the basic data environment provided by auxiliary management function.

\section{SYSTEM DESIGN}

To facilitate user and system management and expansion, the object-oriented modular design approach is used to develop the system in this paper. It includes analysis and estimates module. Statistics and construction cost module, system management module, and the system structure are shown in Figure 1. Each functional module with specific function is independently developed and interlinked through the process management module, and this is conducive to system management and expansion.
To facilitate user operations, dialog control language (DCL) is used to design user management interface in this paper. According to the function setting, the system includes a multi-level user interface. The main interface is used to guide user to select the basic function. Analysis and estimates of length, area and volume, statistics and construction cost and system management are achieved in sub-interfaces. The link between parameters database and the analysis and estimates module and the link between parameters database are established with the process management module. Thus, the data flow of the system is realized. The system detailed use case analysis is shown in Figure 2. In this use case analysis, the engineering staff and the system administrators are as the core.

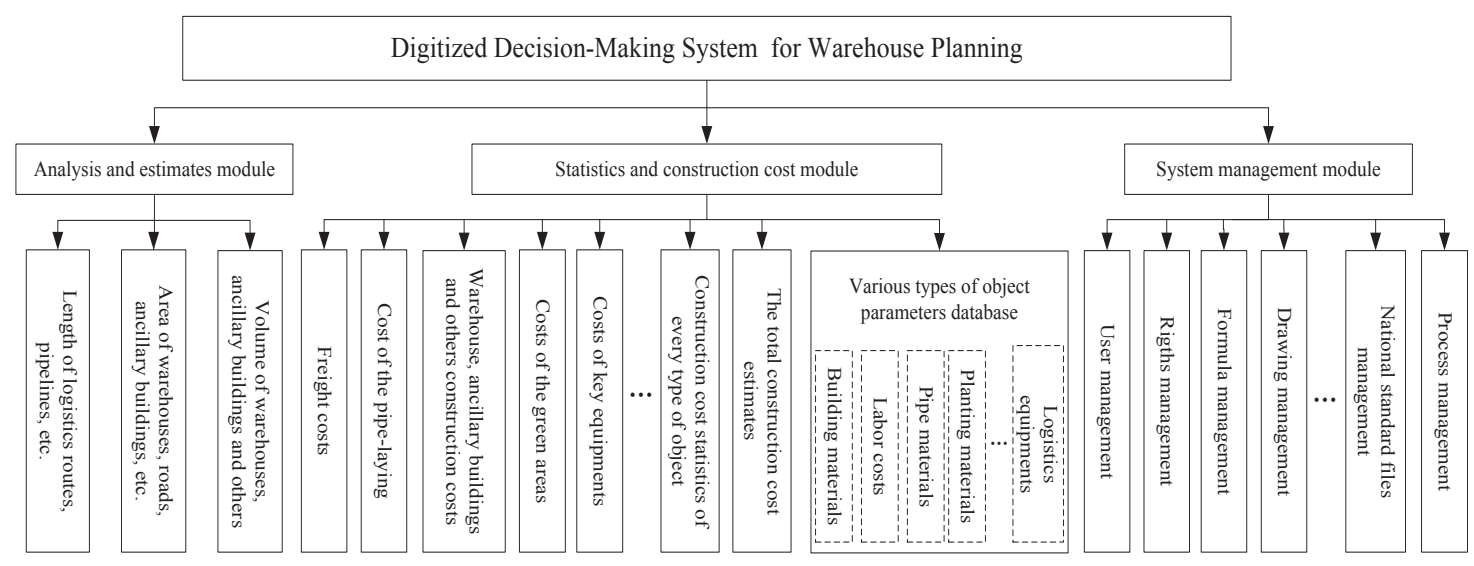

Figure 1. Digitized storage planning decision-making system structure

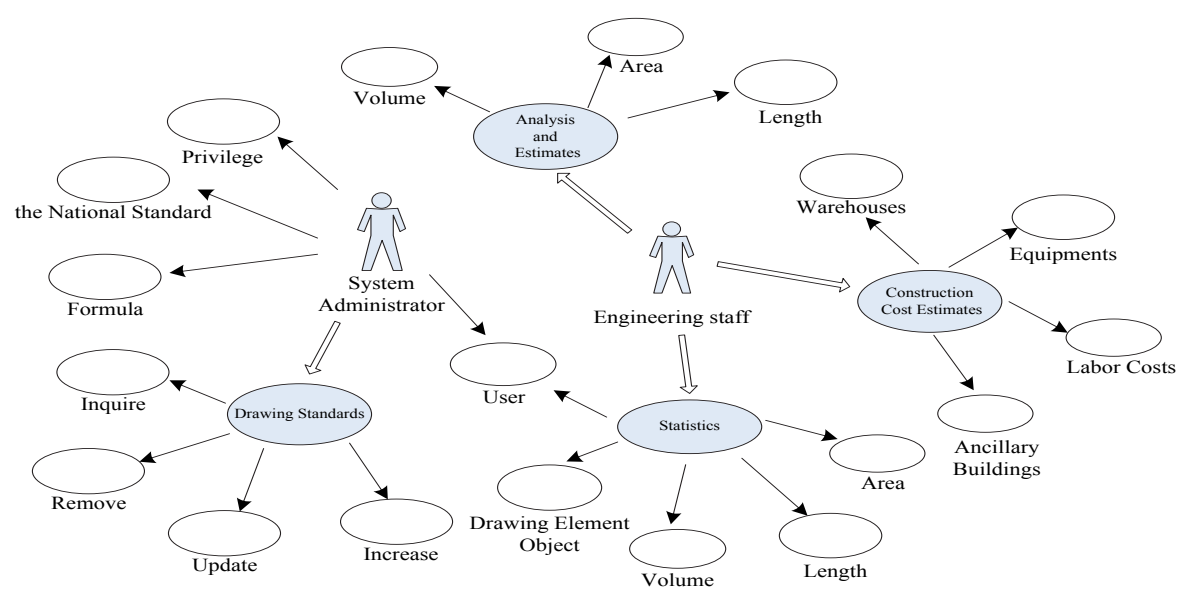

Figure 2. The digitized storage planning decision-making system use case analysis

\section{THE SYSTEM FUNCTION MODEL}

The module design method is used to construct master function modules, which includes the analysis and estimates module, the statistics and construction cost module and the system management module.

\section{A. Analysis and estimates module}

To accurately calculate area and volume for warehouses or ancillary buildings and length for logistics routes, roads and pipelines, through the abstract extraction of every estimates algorithm, the common sub- modules and the special sub-modules are formed separately to meet general and special analysis and estimates requirements and all the algorithm sub-modules are integrated to form the analysis and estimates module. In the process of analysis and calculation, its drawing element object is extracted and the parameters are set by user according to the object type and standards. Then the module calls the specific analysis and estimates programs to estimate its geometric dimensions, with several middle parts of analysis and estimates the ultimate estimates results are obtained and inserted into the SQL database finally. 
During the process of warehouse structure analysis and estimates, a large number of parameters and national standards and industry standards are involved, these parameters and data of national standards and industry standards are often the types of graphs, charts, numeric and character. In the traditional way, the parameters are obtained through repeated query of the manual and the standards. In this system a special warehouse parameters database is designed to manage parameters. According to the parameter expressions, different digital processing methods are used to deal with warehouse parameters, the SLD file is used to store warehouse parameters in graphs and charts and the system calls the corresponding program to display the warehouse parameters in graphs and charts in the specific area defined by the image component in the system interface [10][11]. Numeric and character design parameters are stored in the specific TXT files or drop-down list component, edit control box and other control components in the system interface. Thus, it is convenient to access and determine warehouse parameters in analysis and estimates process.

In order to reduce the analysis and calculation errors for the analysis and estimates object, the system gets data of the primitive automatically, such as layer, line style, line width, color and control point coordinate. Then it analyzes and calculates the length, area and volume, etc. Specifically, for logistics route, pipe network and the distance between two warehouses, the system abstracts and decomposes the object into points, straight line segments and arc segments firstly. Secondly, the formula LP, formula LL and formula LA are used to calculate the length of them or the distance between a point and a line segment separately, these formulas are:

$$
L_{\mathrm{P}}=\sqrt{\left(x_{1}-x_{2}\right)^{2}+\left(y_{2}-y_{2}\right)^{2}}
$$

In which, $\left(x_{1}, y_{1}\right)$ is coordinate of selected starting point (for example: one end of the selected pipeline). $\left(x_{2}, y_{2}\right)$ is coordinate of selected end point (for example: the other end of the selected pipeline).

$$
L_{\mathrm{L}}=\frac{\left|a x_{1}+b y_{1}+c\right|}{\sqrt{a^{2}+b^{2}}}
$$

In which, $\left(x_{1}, y_{1}\right)$ is coordinate of selected point. $a, b$ and $c$ are the parameters or coefficients of the equation of a selected straight line, the equation is $a x+b y+c=0$.

$$
L_{\mathrm{A}}=\frac{n \pi r}{180}
$$

In which, $r$ is the radius of the selected arc. $n$ is the value of the center angle that is corresponding to the selected $\operatorname{arc}\left(^{\circ}\right)$.

Eventually, the final length result is achieved by the formula $L$. Similarly, the sum of the length of the same class object is also calculated by the formula $L$. the formula $L$ is:

$$
L=\sum L_{\mathrm{P} i}+\sum L_{\mathrm{L} j}+\sum L_{\mathrm{A} k}
$$

In which, $L_{\mathrm{Pi}}$ is the $i$ th calculation result of $L_{\mathrm{P}}, L_{\mathrm{Lj}}$ is the $j$ th calculation result of $L_{\mathrm{L}}, L_{\mathrm{Lk}}$ is the $k$ th calculation result of $L_{\mathrm{A}}$.
For warehouse, route and ancillary building, the system abstracts and decomposes the object into basic geometric shapes, such as rectangular, triangular, fanshaped and regular polygon. Then the formula $S_{\mathrm{R}}$, formula $S_{\mathrm{T}}$ and formula $S_{\mathrm{A}}$ are used to calculate the area of them separately. These formulas are:

$$
S_{\mathrm{R}}=a b
$$

In which, $a$ is the width of the selected rectangular (for example: the width of the selected rectangular warehouse), $b$ is the length of the selected rectangular (for example: the width of the selected rectangular warehouse).

$$
S_{\mathrm{T}}=\frac{1}{2} a h
$$

In which, $a$ is the length of the base of the selected triangle, $b$ is the length of the height of the selected triangle.

$$
S_{\mathrm{A}}=\frac{n \pi r^{2}}{360}
$$

In which, $r$ is the radius of the selected fan-shaped, $n$ is the value of the center angle of the selected fan-shaped $\left({ }^{\circ}\right)$.

$$
S_{\mathrm{P}}=\frac{n a^{2}}{4 \tan \frac{180}{n}}
$$

In which, $n$ is the number of polygon sides, $a$ is the length of the polygon sides.

At last, the final area result can be achieved with the formula S, especially for complex objects (after decomposition). Similarly, the sum of the area of the same class object can also be calculated with the formula $\mathrm{S}$. The formula $\mathrm{S}$ is:

$$
S=\sum S_{\mathrm{R} i}+\sum S_{\mathrm{T} j}+\sum S_{\mathrm{A} k}+\sum S_{\mathrm{Pr}}
$$

In which, $S_{\mathrm{R} i}$ is the $i$ th calculation result of $S_{\mathrm{R}}, S_{\mathrm{T} j}$ is the $j$ th calculation result of $S_{\mathrm{T}}, S_{\mathrm{Ak}}$ is the $k$ th calculation result of $S_{\mathrm{A}}, S_{\mathrm{P} r}$ is the $r$ th calculation result of $S_{\mathrm{P}}$.

In addition, as to the volume of analysis and estimates for warehouses, ancillary buildings and others, because the drawings (.DWG) provides plane figure only, after the attraction and decomposition of the object and according to the data of floor height, number of layers and so on, the system completes the separate analysis and estimates and the summary of volume.

In this paper, Visual LISP is utilized to develop the analysis and estimates module and its implementation process [12], as shown in Figure 3.

\section{B. Statistics and construction cost module}

To meet the requirements of engineering staff to take data analysis and statistics, a special SQL database is developed to manage all data. According to the analysis of the system function model, the database consists of four information tables: user information table, drawing element table, legend attributes table as well as attributes description table. The active data objects 
(ADO)technology extended object model used by data access object (DAO)and remote data objects (RDO)contained fewer objects but more properties, methods and events of objects and improved the scalability of the program. So in this system, ADO technology is used to link the user management interface and the system database. The system queries statistics, edit and updates the database only by calling the ADO object model and using open, move, update and other methods of the object [10], as shown in Figure 4.

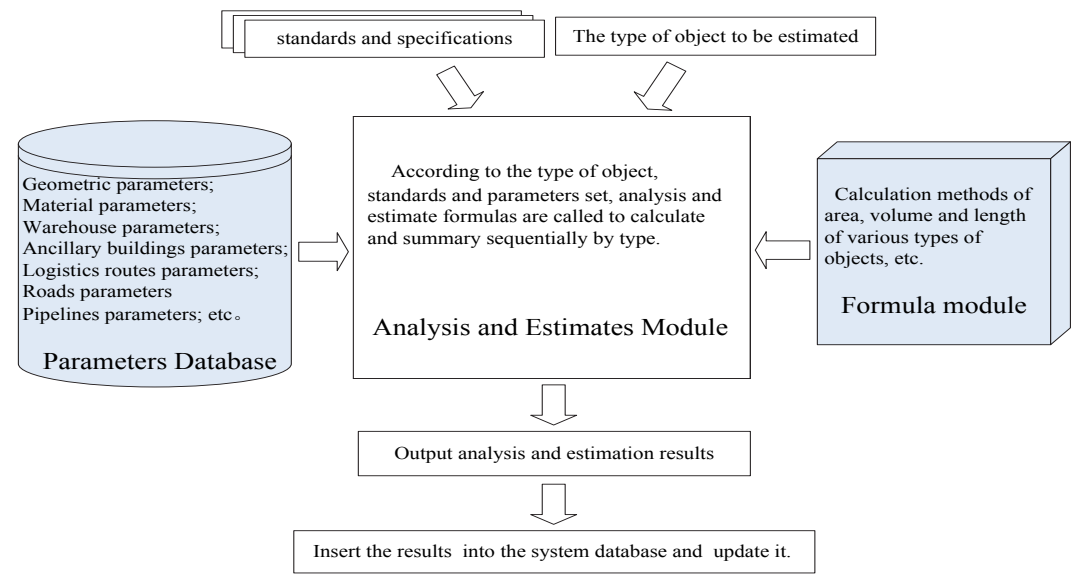

Figure 3. Analysis and estimates module implementation process

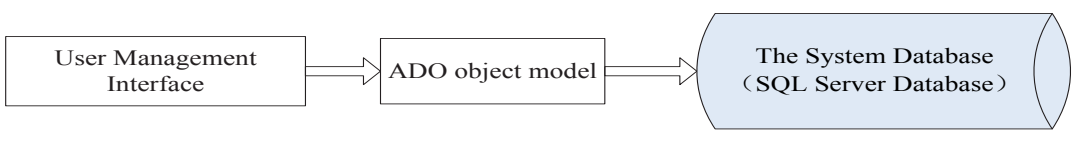

Figure 4. Database interface

In the system, the main objects of queries and statistics are data of attribute, area, volume and length of specified drawing element. Based on these, the dynamic association between the database and the analysis and estimates module achieves real-time database insert and update, the dynamic association between the database and the statistics and construction cost module completes the calculation of warehouse construction cost. In addition, with the user management interface, users are supported to add, delete or edit the standards data of drawing layers of the warehouse DWG files. The construction cost module is developed with Visual LISP program, each algorithm sub-module is developed for a special type of building facilities and all algorithm sub-modules are integrated together to form the total construction cost module. According to construction area or volume of the specified object and the parameters of material, period and others set, with parametric analysis and calculation the module can estimate the construction cost [13]. Finally, the statistical estimates of construction cost for the whole warehouse area is achieved.

\section{System management module}

The system management module is designed for user and rights management, formula management, drawing standards and GB management, etc. The user management contains creation of new user, division of user groups, settings of the roles and permission. According to roles of users, the rights management function assigns user permission. The drawing standards management include: drawing standard query, modification of drawing standards and add or deletion of drawing standards. In addition, the functional properties of different types of drawings and the standards of various types of lines can be stored in SQL database by it. Consequently, the system management module is specially designed to provide basic data environment for the normal operation of the analysis and estimates module and the statistics and construction cost module.

\section{THE STRUCTURE AND IMPLEMENTATION OF THE SYSTEM}

AutoCAD software widely used in the field of engineering drawings, as well as the Visual LISP language is simple and easy to program. Thus the digitized decision-making system of warehouse planning is designed based on Client/Server $(\mathrm{C} / \mathrm{S})$ architecture of Visual LISP, as shown in Figure 5. The driver process of the system management interface established with Visual LISP program is shown in Figure 6.

It is a 3-tier architecture: DCL for user management interface tier, Visual LISP for business logic tier and SQL Server for data storage tier. In AutoCAD through menu items or DCL dialog boxes called by LISP functions, the user management interface meets the demands of user interaction to achieve analysis and estimates, statistics and cost calculation and the system management, etc. The business logic tier is the core of the system, which is responsible for responding to user requests coming from the user interface tier and process it, then it returns the results to the user management tier. The business logic tier can also pass the results to the data tier to store and update the database. Because of $\mathrm{C} / \mathrm{S}$ structure the business logic tier is still running on the client, its main business functions include: system management, analysis and estimates, statistics and so on. In addition, it can also make use of LISP program to drive data control language (DCL) dialog box and complete automated processing of drawing elements and SQL database operation. The data 
storage tier is composed of SQL database and graphical database management, the SQL database used to store all resources information and user data mainly provides data services for business logic tier, such as storage of processing results of the application logic tier and the data return retrieved by the application logic tier. The graphics documents are DWG files stored in the digitized decision-making system of warehouse planning.

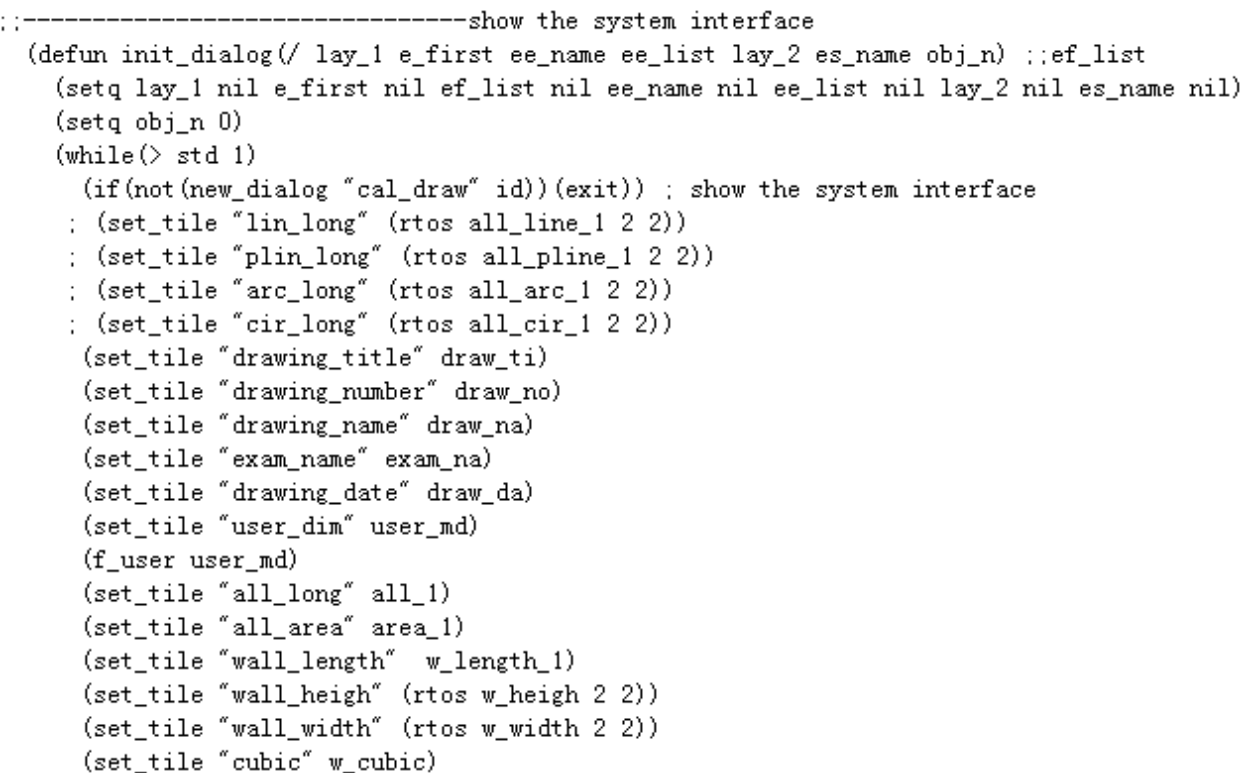

Figure 6 . The driver process of the system management interface

\section{CONCLUSIONS}

Based on Visual LISP and parametric design method, a digitized decision-making system for warehouse planning is developed basing on Visual LISP and SQL Server database. The system interface is friendly and its operation is in line with industry practice. The algorithm programs developed by modular design method not only complete estimating area or volume of various types of warehouses and other buildings, but also complete estimating length of all kinds of pipelines. Thus the system provides the analysis and estimates results as the basis for construction demands. With the analysis and estimates results and according to the building materials, the construction period and other parameters set, the system completes data statistics calls the function module to calculate the construction cost.

Consequently, the system provides a decision-making basis for the warehouse area planning and construction and improves the efficiency of warehouse planning. The approach used in the system can also be a reference to research city or residential district planning and construction.

\section{REFERENCES}

[1] X. X. Bu, Y. Z. Li, "The development of the enterprise logistics management information system based on UML," Int. J. Adv. Comput. Technol., vol. 4, pp. 304-311, 2012. http://dx.doi.org/10.4156/ijact.vol4.issue16.35

[2] G. Y. Xiu, X. H. Chen, "Research on green logistics development at home and abroad," J. Comput., vol. 7, pp. 2765-2772, 2012.

[3] Z. W. Pan, "Course and development of digital design and manufacture," J. Anhui Univ. Technol., vol. 20, pp. 333-339, 2003.

[4] L. Zhai, "Transformation of row material warehouse interior planning for flexible manufacturing enterprises," Logistics Technol., vol. 31, pp. 363-366, 2012.
[5] W. L. Du, "Evaluation system for simulation model of city logistics planning," J. Convergence Inf. Technol., vol. 7, pp. 169176, 2012. http://dx.doi.org/10.4156/jcit.vol7.issue8.19

[6] M. Fogel, N. Burkhart, H. Ren, J. Schiff, M. Meng, and K. Goldberg, "Automated tracking of pallets in warehouses: beacon layout and asymmetric ultrasound observation models," Autom Sci. and Eng., pp. 678-685, 2007.

[7] A. Francesco, B. Francesco, C. Ciro, and C. Pasquale, "An approach to control automated warehouse systems," Control Eng. Pract., vol. 13, pp.1223-1241, 2005. http://dx.doi.org/10.1016/ j.conengprac.2004.10.017

[8] Y. H. Xue, H. P. Liu, "Intelligent storage and retrieval systems based on RFID and vision in automated warehouse," J. Networks, vol. 7, pp. 365-369, 2012.

[9] X. Y. Wu, Z. Z. Yuan, S. H. Cao, and Y. H. Li, "Assessment on warehouse and parking-lots layout plan based on simulation," Logistics Technol., vol. 27, pp. 115-119, 2008.

[10] H. Yao, Y. Cao, "Development of a centripetal knuckle bearing performance analysis and parametric drawing system based on autoCAD and VBA," Chem. Eng. Mater. Prop. II, vol. 549, pp. 889-893, 2012.

[11] H. Yao and Y. Cao, "Integrated rolling bearing type selection and parameter-driven graphic resource system," Mater. Sci. Infor. $n$ Technol. I, vol. 532-533, pp. 441-445, 2012.

[12] L. Zheng, T. J. Li, Z. J. Huo, "Design and implementation of the strategic reserve material support decision support system," Military Oper. Res. Syst. Eng, vol. 25, pp.63-66, 2011.

[13] H. Jiang, M. S. Yang, "Logistics center warehouse layout planning," New West, pp. 34-35, 2008.

\section{AUTHORS}

H. Yao, Y. D. Fang, and Y. Cao are with the School of Mechatonic Engineering, Xi'an Technological University, Xi'an, China.

Submitted 01 July 2013. Published as re-submitted by the authors 15 September 2013 . 